\title{
Dynamic Green's functions of a buried point load with applications to drainage
}

\author{
B. Y. Ding \& L. J. Zhu \\ The College of Civil Engineering, \\ Zhejiang University of Technology, China
}

\begin{abstract}
Based on the Biot's equations for poroelastic media and the decoupling of fast and slow longitudinal waves, the authors obtain the fundamental Green's functions in the frequency domain for the solid and fluid phases subjected to a buried point load. The numerical results on drained displacement, drained pore pressure and the flux are presented in this paper via Somigliana's integral and Hankel transformation. As an application, the drained flux due to vibroflotation in ground treatment in saturated soil is discussed in detail.

Keywords: drainage, Green's function, buried point load, saturated soil, dynamic response.
\end{abstract}

\section{Introduction}

Saturated soil is generally described as a poroelastic medium by scientists and engineers [1-6] and thus dynamic theory in a poroelastic medium is important. While Huang and Zhang [7] used the integral transformation to attack the nonaxisymmetric Lamb's problem [7], Philippacopoulos [8] obtained the solutions of the Lamb's problem in a two-phase saturated medium via decomposition of the potential functions. Jin and Liu [9] derived the three-dimensional dynamic response of the poroelastic half-space subjected to a buried point force. In previous studies of poroelastic problems, the drainage issue is ignored. However, water drainage in saturated soil occurs when a shock load is applied. A similar drained phenomenon can also be observed in pile driving and vibroflotation during ground treatment. 
In this paper, by decoupling the fast and slow longitudinal waves in Biot's poroelastic equation, authors derive the corresponding Green's functions of the solid phases and the fluid phases for the saturated half-space subjected to a buried point load in the frequency domain [10-13]. Then by applying drained Somigliana's integral associated with the Hankel transformation $[10,11]$ and ignoring the radial displacement, the drained results from vibroflotation in ground treatment have been obtained, including the drained displacement, drained pore pressure and flux. Representative figures are presented and discussed.

We should point out that the solution obtained does not include irreversible deformation of the soil during vibroflotation in ground treatment and that the radial displacement is also ignored for simplicity. While these two issues will be addressed in the future, the results could be useful in analyzing the dynamic response with drainage in liquefaction, the drained properties of dynamic response for various kinds of foundations in the saturated soil, and the interaction of traffic loading and the roadbed in the saturated soil.

\section{Dynamic equations and solutions}

Biot's dynamic equations of a poroelastic medium under axisymmetric deformation and in the frequency domain can be written as $[8,9]$ :

$$
\begin{gathered}
\mu\left(\nabla^{2} u_{r}-u_{r} / r^{2}\right)+\left(\lambda_{c}+\mu\right) \partial e / \partial r-a M \partial \xi / \partial r=-\rho \omega^{2} u_{r}-\rho_{f} \omega^{2} w_{r}, \\
\mu \nabla^{2} u_{z}+\left(\lambda_{c}+\mu\right) \partial e / \partial z-a M \partial \xi / \partial z=\rho \omega^{2} u_{z}+\rho_{f} \omega^{2} w_{z}, \\
a M \partial e / \partial r /-M \partial \xi / \partial r=\rho_{f} \omega^{2} u_{z}+\gamma \omega^{2} w_{z}, \\
a M \partial e / \partial r /-M \partial \xi / \partial r /=\rho_{f} \omega^{2} u_{z}+\gamma \omega^{2} w_{z} \\
\lambda_{\mathrm{c}}=\lambda+\alpha^{2} M \\
\gamma=\left[-\mathrm{i} \beta_{0}^{2}+\omega \kappa\left(\rho_{a}+\beta_{0} \rho_{f}\right)\right] /\left(\beta_{0}^{2} \omega \kappa\right),
\end{gathered}
$$

where $\lambda$ and $\mu$ are the Lamé coefficients; $\alpha$ and $M$ the parameters introduced by Biot in his investigation of the two-phase saturated medium; $\rho$ and $\rho_{f}$ the mass density of the two-phase saturated medium and the fluid phase respectively, $\rho_{a}$ the apparent mass density of the two-phase saturated medium; $u_{r}$ and $u_{z}$ the frequency-domain displacement components of solid phase in radial and vertical direction respectively, $w_{r}$ and $w_{z}$ the frequency-domain displacement components of the fluid phase relative to the solid phase in radial and vertical direction respectively; $\beta_{0}$ the porosity; $\kappa=\eta_{0} / k_{d}$ with $\eta_{0}$ being the viscosity coefficient and $k_{d}$ the dynamic permeability coefficient; $\omega$ the 
circular frequency; $\mathrm{i}$ the imaginary unit; $\xi$ the volumetric strain of the fluid phase; and $e$ is the volumetric strain of the solid phase.

It is noted that $\mathbf{u}_{1}$ and $\mathbf{u}_{2}$ can be defined as the displacement vectors of the fast and slow longitudinal wave respectively due to formulas $\mathbf{u}_{1}+\mathbf{u}_{2}=\mathbf{u}$ and $\xi_{1} \mathbf{u}_{1}+\xi_{2} \mathbf{u}_{2}=\mathbf{w}$ with the decoupling (dynamic) coefficient being $\xi_{i}=\left[\lambda+2 \mu+\alpha^{2} M-\rho\left(\alpha_{i}\right)^{2}\right] /\left[\rho_{f}\left(\alpha_{i}\right)^{2}-\alpha M\right] \quad(i=1,2)$ and $\mathbf{w}$ being the displacement vector of the fluid phase relative to the solid phase. The detailed definitions and explanations can be found in references $[3,12,13]$. Thus, for the volumetric strain of the solid phase, we have $e_{i}=\partial u_{r i} / \partial r+u_{r i} / r+\partial u_{z i} / \partial z$, and for the volumetric strain of the fluid phase $\xi=\xi_{i} \partial u_{r i} / \partial r+\xi_{i} u_{r i} / r+\xi_{i} \partial u_{z i} / \partial z$. It should be noted that summation convention for repeated subscript $i$ in the expression for the volumetric body strain of the fluid phase is implied.

From Eqn. (1-1) + Eqn. (1-2), we can find the expressions for the divergence of the displacement in the solid phase as follows:

$$
\begin{aligned}
& \alpha_{1}^{2} \nabla^{2} e^{(1)}=-\omega^{2} e^{(1)}, \\
& \alpha_{2}^{2} \nabla^{2} e^{(2)}=-\omega^{2} e^{(2)} .
\end{aligned}
$$

where $\alpha_{1}$ and $\alpha_{2}$ are the velocities of fast and slow longitudinal waves respectively; $e^{(1)}$ and $e^{(2)}$ the divergences of the displacements of the fast and slow longitudinal wave respectively; and $\nabla^{2}$ is a Laplace's operator. We define $f^{v}(p)$ the $v$-order Hankel's transformation with respect to the function $f(r)$. Then, by applying the zero-order Hankel transformation to Eqns (4-1) and (4-2), we can obtain the following two equations:

$$
\begin{aligned}
& \left(\lambda_{c}+2 \mu+\alpha M \xi_{1}\right)\left[\left(\frac{d^{2}}{d z^{2}}-p^{2}\right)+\frac{\left(\rho+\rho_{f} \xi_{1}\right) \omega^{2}}{\lambda_{c}+2 \mu+\alpha M \xi_{1}}\right] e^{(1)}=0, \\
& \left(\lambda_{c}+2 \mu+\alpha M \xi_{2}\right)\left[\left(\frac{d^{2}}{d z^{2}}-p^{2}\right)+\frac{\left(\rho+\rho_{f} \xi_{2}\right) \omega^{2}}{\lambda_{c}+2 \mu+\alpha M \xi_{2}}\right] e^{(2)}=0 .
\end{aligned}
$$

Then, from [14], the solution of Eqns. (5-1) and (5-2) can be written as follows:

$$
\begin{gathered}
e^{(1)}=A_{1}^{(1)} e^{-a^{(1)} z}+A_{2}^{(1)} e^{a^{(1)} z} \\
e^{(2)}=A_{1}^{(2)} e^{-a^{(2)} z}+A_{2}^{(2)} e^{a^{(1)} z}
\end{gathered}
$$

where $_{a^{(1)}}=\sqrt{p^{2}-K_{\alpha_{1}}^{2}}, \quad a^{(2)}=\sqrt{p^{2}-K_{\alpha_{2}}^{2}} ; K_{\alpha_{1}}$ and $K_{\alpha_{2}}$ are the fast and slow longitudinal wave numbers respectively. The superscripts (1) and (2) are used to indicate the fast and slow longitudinal waves respectively. In addition, we have [13]: 


$$
K_{\alpha_{1}}^{2}=\frac{\left(\rho+\rho_{f} \xi_{1}\right) \omega^{2}}{\lambda_{c}+2 \mu+\alpha M \xi_{1}}, \quad K_{\alpha_{2}}^{2}=\frac{\left(\rho+\rho_{f} \xi_{2}\right) \omega^{2}}{\lambda_{c}+2 \mu+\alpha M \xi_{2}} .
$$

Furthermore, applying the zero-order Hankel's transformation to the dynamic Eqn. (1-2) in vertical direction, we obtain:

$$
\mu \nabla^{2} u_{z}^{0}+\rho_{f} \omega^{2} w_{z}+\rho \omega^{2} u_{z}^{0}=-\left(\lambda_{c}+\mu\right) \partial e^{0} / \partial z /+a M \partial \xi / \partial z
$$

Noting $\xi_{1} \mathbf{u}_{1}+\xi_{2} \mathbf{u}_{2}=\mathbf{w}$, we readily have the following formula

$$
\begin{aligned}
\mu \nabla^{2} u_{z}^{0}+\rho_{f} \omega^{2}\left(\xi_{1} u_{z 1}^{0}+\xi_{2} u_{z 2}^{0}\right)+\rho \omega^{2} u_{z}^{0} & =-\left(\lambda_{c}+\mu\right) \partial e^{0} / \partial z / \\
& +a M \partial\left(\xi_{1} e^{(1) 0}+\xi_{2} e^{(2) 0}\right) / \partial z
\end{aligned}
$$

Thus,

$$
\begin{aligned}
& \left(\mu \nabla^{2}+\rho_{f} \omega^{2} \xi_{1}+\rho \omega^{2}\right) u_{z 1}^{0}+\left(\mu \nabla^{2}+\rho_{f} \omega^{2} \xi_{2}+\rho \omega^{2}\right) u_{z 2}^{0} \\
& \quad=-\left(\lambda_{c}+\mu+a M \xi_{1}\right) \partial e^{(1) 0} / \partial z-\left(\lambda_{c}+\mu+a M \xi_{2}\right) \partial e^{(2) 0} / \partial z
\end{aligned}
$$

From Eqn. (8-3) and also considering Eqn. (6), we obtain [14, 15]

$$
\begin{aligned}
u_{z}^{0}= & -a^{(1)}\left(A_{1}^{(1)} e^{-a^{(1)} z}+A_{2}^{(1)} e^{a^{(1)} z}\right)+a^{(2)}\left(A_{1}^{(2)} e^{-a^{(2)} z}+A_{2}^{(2)} e^{a^{(2)} z}\right), \\
& +p^{2}\left(R_{1} e^{-b z}+R_{2} e^{b z}\right)
\end{aligned}
$$

where $b=\sqrt{p^{2}-K_{\beta}^{2}}, K_{\beta}^{2}=\left[\left(\rho-\rho_{f}^{2} / \gamma\right) \omega^{2}\right] / \mu$ with $K_{\beta}$ the transverse wave number; $R_{1}$ and $R_{2}$ are the arbitrary functions corresponding to $p$. Based on the relation $u_{r}^{1}=\left(e^{0}-d u_{z}^{0} / d z\right) / p$, we obtain $[14,15]$ :

$$
\begin{aligned}
u_{r}^{1}= & -p\left(A_{1}^{(1)} e^{-a^{(1)} z}+A_{2}^{(1)} e^{a^{(1)} z}\right)+p\left(A_{1}^{(2)} e^{-a^{(2)} z}+A_{2}^{(2)} e^{a^{(2)} z}\right) . \\
& +p \mathrm{i}\left(R_{1} e^{-b z}+R_{2} e^{b z}\right)
\end{aligned}
$$

Thus, the displacement of the fluid phase can be derived as

$$
\begin{aligned}
w_{r}^{1}= & -p \xi_{1}\left(A_{1}^{(1)} e^{-a^{(1)} z}+A_{2}^{(1)} e^{a^{(1)} z}\right)+p \xi_{2}\left(A_{1}^{(2)} e^{-a^{(2)} z}+A_{2}^{(2)} e^{a^{(2)} z}\right) \\
& +\xi_{3} p \mathrm{i}\left(R_{1} e^{-b z}+R_{2} e^{b z}\right)
\end{aligned}
$$

where $\xi_{3}=-\rho_{f} / \gamma[8]$. The corresponding expressions of the effective stress and pore pressure can be deduced as follows:

From $t_{z w w}=\mu\left(\partial u_{r} / \partial z+\partial u_{z} / \partial r\right)$, we obtain

$$
\begin{aligned}
t_{z r w}^{1}=2 \mu p a^{(1)}( & \left.A_{1}^{(1)} e^{-a^{(1)} z}+A_{2}^{(1)} e^{a^{(1)} z}\right)+2 \mu p a^{(2)}\left(A_{1}^{(2)} e^{-a^{(2) z}}\right. \\
& \left.+A_{2}^{(2)} e^{a^{(2)} z}\right)-\mu p\left(p^{2}+b^{2}\right)\left(R_{1} e^{-b z}+R_{2} e^{b z}\right)
\end{aligned}
$$

From Darcy's law $P_{w}=-\alpha M e-M \xi$, we obtain

$$
\begin{aligned}
P_{w}^{0}=-\left(\alpha+\xi_{1}\right) M k_{\alpha 1}{ }^{2}\left(A_{1}^{(1)} e^{-a^{(1)} z}+A_{2}^{(1)} e^{a^{(1) z}}\right) \\
-\left(\alpha+\xi_{2}\right) M k_{\alpha 2}{ }^{2}\left(A_{1}^{(2)} e^{-a^{(2)} z}+A_{2}^{(2)} e^{a^{(2)} z}\right) .
\end{aligned}
$$


From $\sigma_{z w}=\lambda e+2 \mu \partial u_{z} / \partial z$, we obtain

$$
\begin{aligned}
\sigma_{z w}^{0}= & {\left[(\lambda+2 \mu)\left(a^{(1)}\right)^{2}-\lambda p^{2}\right]\left(A_{1}^{(1)} e^{-a^{(1)} z}+A_{2}^{(1)} e^{a^{(1)} z}\right) } \\
& +\left[(\lambda+2 \mu)\left(a^{(2)}\right)^{2}-\lambda p^{2}\right]\left(A_{1}^{(2)} e^{-a^{(2)} z}+A_{2}^{(2)} e^{a^{(2) z} z}\right) . \\
& -2 \mu p^{2} b\left(R_{1} e^{-b z}+R_{2} e^{b z}\right)
\end{aligned}
$$

where $t_{z r w}$ is the effective shear stress corresponding to the stress component $t_{z r}$, $P_{w}$ is the pore pressure and $\sigma_{z w}$ denotes the effective normal stress in the z-direction (vertical).

\section{Boundary and interface conditions}

Considering a saturated poroelastic half-space, we divide it into two parts as shown in Fig. 1: part $\mathrm{A}$ is in $\left(0 \leq z \leq z^{\prime}\right)$ and part $\mathrm{B}$ in $\left(z^{\prime}<z<\infty\right)$. The superscripts $\mathrm{A}$ and $\mathrm{B}$ are used to distinguish the upper and lower parts respectively. The displacement and stress conditions in the saturated poroelastic half-space can be written as

$$
\begin{gathered}
\sigma_{z w}^{\mathrm{A}}(r, 0)=0, t_{z r w}^{\mathrm{A}}(r, 0)=0, \\
p_{z w}^{\mathrm{A}}(r, 0)=0, \\
u_{z}^{\mathrm{A}}\left(r, z^{\prime}\right)-u_{z}^{\mathrm{B}}\left(r, z^{\prime}\right)=0, \\
u_{r}^{\mathrm{A}}\left(r, z^{\prime}\right)-u_{r}^{\mathrm{B}}\left(r, z^{\prime}\right)=0 \\
p_{z w}^{\mathrm{A}}\left(r, z^{\prime}\right)-p_{z w}^{\mathrm{B}}\left(r, z^{\prime}\right)=0, \\
\sigma_{z w}^{\mathrm{A}}\left(r, z^{\prime}\right)-\sigma_{z w}^{\mathrm{B}}\left(r, z^{\prime}\right)=F_{0} \delta(r) / 2 \pi r \\
t_{z r w}^{\mathrm{A}}\left(r, z^{\prime}\right)-t_{z r w}^{\mathrm{B}}\left(r, z^{\prime}\right)=0, \\
w_{z}^{\mathrm{A}}\left(r, z^{\prime}\right)-w_{z}^{\mathrm{B}}\left(r, z^{\prime}\right)=0 .
\end{gathered}
$$

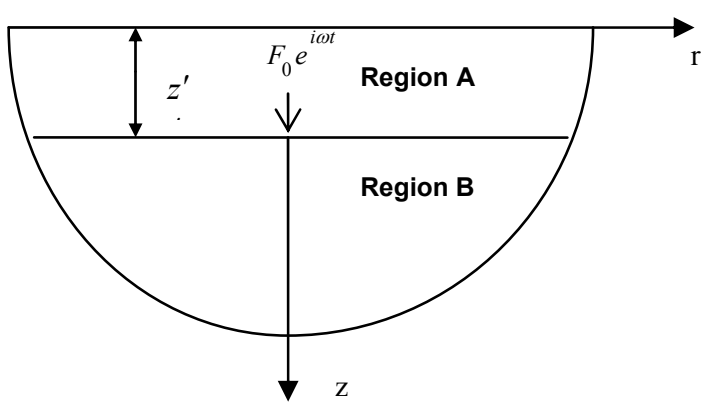

Figure 1: The system of saturated half-space. 
The radiation condition of the waves can be expressed as $A_{2}^{(1) B}=A_{2}^{(2) B}=R_{2}^{B}=0$.

For the vertical buried concentrated force of $F_{0} \delta(r) / 2 \pi r$, we can obtain:

$$
\begin{aligned}
& A_{1}^{(1) \mathrm{A}}= F\left\{\left(\alpha+\xi_{2}\right) k_{\alpha_{2}}{ }^{2} f_{1} e^{-a^{(1)} z^{\prime}}-8 a^{(2)} \mu p^{2} b(\alpha\right. \\
&\left.+\xi_{2}\right) k_{\alpha_{2}}{ }^{2}\left(\alpha+\xi_{1}\right) k_{\alpha_{1}}{ }^{2} e^{-a^{(2)} z^{\prime}}+4 \mu p^{2}\left(p^{2}+b^{2}\right)(\alpha \\
&\left.\left.+\xi_{2}\right) k_{\alpha_{2}}{ }^{2}\left[\left(\alpha+\xi_{1}\right) k_{\alpha_{1}}{ }^{2}-\left(\alpha+\xi_{2}\right) k_{\alpha_{2}}{ }^{2}\right] e^{-b z^{\prime}}\right\} / 4 \pi Y f \\
& A_{2}^{(1) \mathrm{A}}=F\left[\left(\alpha+\xi_{2}\right) k_{\alpha_{2}}{ }^{2} e^{-a^{(1)} z^{\prime}}\right] / 4 \pi Y \\
& A_{1}^{(2) \mathrm{A}}= F\left\{-8 a^{(1)} \mu k^{2} b\left(\alpha+\xi_{1}\right) k_{\alpha_{1}}{ }^{2}\left(\alpha+\xi_{2}\right) k_{\alpha_{2}}{ }^{2} e^{-a^{(1)} z^{\prime}}\right. \\
&+\left(\alpha+\xi_{1}\right) k_{\alpha_{1}}{ }^{2} f_{2} e^{-a^{(2)} z^{\prime}}-4 \mu k^{2}\left(k^{2}+b^{2}\right)(\alpha \\
&\left.\left.+\xi_{1}\right) k_{\alpha_{1}}{ }^{2}\left[\left(\alpha+\xi_{1}\right) k_{\alpha_{1}}{ }^{2}-\left(\alpha+\xi_{2}\right) k_{\alpha_{2}}{ }^{2}\right] e^{-b z^{\prime}}\right\} / 4 \pi Y f \\
& A_{2}^{(2) \mathrm{A}}=-F\left[\left(\alpha+\xi_{1}\right) k_{\alpha_{1}}{ }^{2} e^{-a^{(2)} z^{\prime}}\right] / 4 \pi Y \\
& R_{1}^{A}= F\left\{-4 a^{(1)} b\left(\alpha+\xi_{2}\right) k_{\alpha_{2}}{ }^{2}\left[h_{2}\left(\alpha+\xi_{1}\right) k_{\alpha_{1}}{ }^{2}\right.\right. \\
&-\left.h_{1}\left(\alpha+\xi_{2}\right) k_{\alpha_{2}}{ }^{2}\right] e^{-a^{(1)} z^{\prime}}+4 a^{(2)} b\left(\alpha+\xi_{1}\right) k_{\alpha_{1}}{ }^{2}\left[h_{2}(\alpha\right. \\
&+\left.\left.\xi_{1}\right) k_{\alpha_{1}}{ }^{2}-h_{1}\left(\alpha+\xi_{2}\right) k_{\alpha_{2}}{ }^{2}\right] e^{-a^{(2)} z^{\prime}} \\
&- {\left[\left(\alpha+\xi_{1}\right) k_{\alpha_{1}}{ }^{2}-\left(\alpha+\xi_{2}\right) k_{\alpha_{2}}{ }^{2}\right] f_{3} e^{-b z^{\prime}} / 4 \pi Y f b } \\
& R_{1}^{B}=-F\left[\left(\alpha+\xi_{1}\right) k_{\alpha_{1}}{ }^{2}-\left(\alpha+\xi_{2}\right) k_{\alpha_{2}}{ }^{2}\right] e^{-b z^{\prime}} / 4 \pi Y b \\
& A_{1}^{(1) \mathrm{B}}=A_{1}^{(1) \mathrm{A}}-A_{2}^{(1) \mathrm{A}} e^{2 a^{(1)} z^{\prime}}, \\
& A_{1}^{(2) \mathrm{B}}=A_{1}^{(2) \mathrm{A}}-A_{2}^{(2) \mathrm{A}} e^{2 a^{(2)} z^{\prime}} \\
& R_{1}{ }^{B}=R_{1}^{A}-R_{2}^{A} e^{2 b z^{\prime}}
\end{aligned}
$$

where

$$
\begin{aligned}
Y= & \left(2 \mu p^{2}-h_{1}\right)\left[\left(\alpha+\xi_{1}\right) k_{\alpha_{1}}{ }^{2}-\left(\alpha+\xi_{2}\right) k_{\alpha_{2}}{ }^{2}\right] \\
& -\left(h_{2}-h_{1}\right)\left(\alpha+\xi_{1}\right) k_{\alpha_{1}}{ }^{2} \\
f= & -\left(\alpha+\xi_{2}\right) k_{\alpha_{2}}^{2}\left[h_{1}\left(p^{2}+b^{2}\right)-4 a^{(1)} \mu p^{2} b\right] \\
& +\left(\alpha+\xi_{1}\right) k_{\alpha_{1}}^{2}\left[h_{2}\left(p^{2}+b^{2}\right)-4 a^{(2)} \mu p^{2} b\right] \\
f_{1}= & \left(\alpha+\xi_{2}\right) k_{\alpha_{2}}{ }^{2}\left[h_{1}\left(p^{2}+b^{2}\right)+4 a^{(1)} \mu p^{2} b\right] \\
& -\left(\alpha+\xi_{1}\right) k_{\alpha_{1}}{ }^{2}\left[h_{2}\left(p^{2}+b^{2}\right)-4 a^{(2)} \mu p^{2} b\right] \\
& f_{2}=-\left(\alpha+\xi_{2}\right) k_{\alpha_{2}}{ }^{2}\left[h_{1}\left(p^{2}+b^{2}\right)-4 a^{(1)} \mu p^{2} b\right] \\
& +\left(\alpha+\xi_{1}\right) k_{\alpha_{1}}{ }^{2}\left[h_{2}\left(p^{2}+b^{2}\right)+4 a^{(2)} \mu p^{2} b\right]
\end{aligned}
$$




$$
\begin{gathered}
f_{3}=-\left(\alpha+\xi_{2}\right) k_{\alpha_{2}}{ }^{2}\left[h_{1}\left(p^{2}+b^{2}\right)+4 a^{(1)} \mu p^{2} b\right] \\
+\left(\alpha+\xi_{1}\right) k_{\alpha_{1}}{ }^{2}\left[h_{2}\left(p^{2}+b^{2}\right)+4 a^{(2)} \mu p^{2} b\right] \\
h_{1}=(\lambda+2 \mu)\left(a^{(1)}\right)^{2}-\lambda p^{2}, h_{2}=(\lambda+2 \mu)\left(a^{(2)}\right)^{2}-\lambda p^{2}
\end{gathered}
$$

\section{The Green's functions and the Somigliana's integration}

By substituting Eqns. (13)-(21) into Eqns. (9)-(11), we can determine the nine coefficients as in $[3,12]$ and [13]. Hence, we eventually obtain the Green's functions for a saturated poroelastic medium. Then, by the inverse Hankel's transform, the Green's functions can be expressed as:

For the solid phase

$$
\begin{aligned}
G_{z z}= & \int_{0}^{\infty}\left[-a^{(1)}\left(A_{1}^{(1)} e^{-a^{(1)} z}+A_{2}^{(1)} e^{a^{(1)} z}\right)-a^{(2)}\left(A_{1}^{(2)} e^{-a^{(2)} z},\right.\right. \\
& \left.\left.+A_{2}^{(2)} e^{a^{(2)} z}\right)+k^{2}\left(R_{1} e^{-b z}+R_{2} e^{b z}\right)\right] J_{0}(p r) p \mathrm{~d} p \\
G_{r z} & =\int_{0}^{\infty}\left[-p\left(A_{1}^{(1)} e^{-a^{(1)} z}+A_{2}^{(1)} e^{a^{(1)} z}\right)-p\left(A_{1}^{(2)} e^{-a^{(2)} z}\right.\right. \\
& \left.\left.+A_{2}^{(2)} e^{a^{(2)} z}\right)+p b\left(R_{1} e^{-b z}+R_{2} e^{b z}\right)\right] J_{1}(p r) p \mathrm{~d} p
\end{aligned}
$$

For the fluid phase

$$
\begin{aligned}
G_{4 z}= & \int_{0}^{\infty}\left[-\left(\alpha+\xi_{1}\right) M k_{\alpha 1}^{2}\left(A_{1}^{(1)} e^{-a^{(1)} z}+A_{2}^{(1)} e^{a^{(1)} z}\right)\right. \\
& \left.-\left(\alpha+\xi_{2}\right) M k_{\alpha 2}{ }^{2}\left(A_{1}^{(2)} e^{-a^{(2)} z}+A_{2}^{(2)} e^{a^{(2)} z}\right)\right] J_{0}(p r) p \mathrm{~d} p \\
G_{z 4}= & \frac{1}{i \omega} \int_{0}^{\infty}\left[-\left(\alpha+\xi_{1}\right) M k_{\alpha 1}^{2}\left(A_{1}^{(1)} e^{-a^{(1)} z}+A_{2}^{(1)} e^{a^{(1)} z}\right)\right. \\
& \left.-\left(\alpha+\xi_{2}\right) M k_{\alpha 2}{ }^{2}\left(A_{1}^{(2)} e^{-a^{(2)} z}+A_{2}^{(2)} e^{a^{(2)} z}\right)\right] J_{0}(p r) p \mathrm{~d} p \\
& G_{44}=\frac{1}{i \omega} \int_{0}^{\infty}\left[\left(\alpha+\xi_{1}\right)^{2} M^{2} k_{\alpha 1}{ }^{2}\left(A_{1}^{(1)} e^{-a^{(1)} z}+A_{2}^{(1)} e^{a^{(1)} z}\right)\right. \\
+ & \left.\left(\alpha+\xi_{2}\right)^{2} M^{2} k_{\alpha 2}{ }^{2}\left(A_{1}^{(2)} e^{-a^{(2)} z}+A_{2}^{(2)} e^{a^{(2)} z}\right)\right] J_{0}(p r) p \mathrm{~d} p
\end{aligned}
$$

Since in the solutions in this paper, the displacement in the radial direction is ignored, the drained displacement $u_{z}^{*}$ can be expressed via Somigliana's integration, as in $[10,16]$ :

$$
\begin{aligned}
& c_{s} u_{z}^{*}(\mathbf{r})=\iint_{S}\left[t_{j}\left(\mathbf{r}_{0}\right) \dot{G}_{z j}\left(\mathbf{r}-\mathbf{r}_{0}\right)-\dot{\sigma}_{j k}\left(\mathbf{r}-\mathbf{r}_{0}\right) u_{j}^{*}\left(\mathbf{r}_{0}\right)\right. \\
& \left.\quad+p\left(\mathbf{r}_{0}\right) \xi_{i} G_{z j(i)}\left(\mathbf{r}-\mathbf{r}_{0}\right)-G_{4 z}\left(\mathbf{r}-\mathbf{r}_{0}\right) \xi_{i} u_{z(i)}^{*}\left(\mathbf{r}_{0}\right)\right] \mathrm{d} S
\end{aligned}
$$

where $\mathbf{r}$ and $\mathbf{r}_{0}$ are the coordinates of the field and source points respectively, the dot above a variable denotes the differentiation with respect to time.

Similarly, the drained pore pressure is: 


$$
\begin{aligned}
& c_{s} P_{w}(\mathbf{r})=\iint_{s}\left[t_{j}\left(\mathbf{r}_{\mathbf{0}}\right) \dot{G}_{j 4}\left(\mathbf{r}-\mathbf{r}_{\mathbf{0}}\right)-\dot{P}_{j}\left(\mathbf{r}-\mathbf{r}_{\mathbf{0}}\right) u_{j}^{*}\left(\mathbf{r}_{\mathbf{0}}\right)\right. \\
& \left.\quad+p_{w}\left(\mathbf{r}_{\mathbf{0}}\right) \xi_{i} G_{j 4(i)}\left(\mathbf{r}-\mathbf{r}_{\mathbf{0}}\right)-G_{44}\left(\mathbf{r}-\mathbf{r}_{\mathbf{0}}\right) \xi_{i} u_{i}^{*}\left(\mathbf{r}_{\mathbf{0}}\right)\right] \mathrm{d} S
\end{aligned}
$$

The flux of drainage is [10]:

$$
\begin{aligned}
c_{s} q_{j}^{*}(\mathbf{r})=\iint[ & t_{k}\left(\mathbf{r}_{\mathbf{0}}\right) g_{j k}^{*}\left(\mathbf{r}-\mathbf{r}_{\mathbf{0}}\right)-h_{j k}^{*}\left(\mathbf{r}-\mathbf{r}_{\mathbf{0}}\right) u_{k}^{*}\left(\mathbf{r}_{\mathbf{0}}\right) \\
& \left.\quad-p_{w}\left(\mathbf{r}_{\mathbf{0}}\right) g_{j}^{*}\left(\mathbf{r}-\mathbf{r}_{\mathbf{0}}\right)-h_{j}^{*}\left(\mathbf{r}-\mathbf{r}_{\mathbf{0}}\right) q^{*}\left(\mathbf{r}_{\mathbf{0}}\right)\right] \mathrm{d} s
\end{aligned}
$$

where

$$
\begin{aligned}
g_{i j}^{*}= & \beta_{0}\left(G_{i 4, j} / \rho_{f}+\dot{G}_{i j}\right), h_{j}^{*}=\beta_{0}\left[\dot{G}_{44, j} /\left(\omega^{2} \rho_{f}\right)-G_{4 j}\right] \\
h_{k j}^{*}= & \beta_{0}\left[\lambda G_{k 4, k} n_{j}+\mu\left(G_{j 4, k}+G_{k 4, j}\right) n_{k}-\alpha G_{44} n_{j}\right]_{, j} / \rho_{f} \\
& +\left[\lambda \dot{G}_{k j, k} n_{j}+\mu\left(\dot{G}_{k j, k}+\dot{G}_{k j, i}\right) n_{k}-\alpha \dot{G}_{4 j} n_{j}\right] \\
g_{j}^{*}= & \left(\beta_{0} / \omega \rho_{f}\right)^{2}\left[\left(G_{44, i}-\omega^{2} \rho_{f} G_{i 4}\right) n_{k}\right]_{, j} \\
& -\left(\beta_{0}{ }^{2} / \omega^{2} \rho_{f}\right)\left[\left(\dot{G}_{4 j, i}-\omega^{2} \rho_{f} \dot{G}_{i j}\right) n_{k}\right]
\end{aligned}
$$

The notations $n_{k}$ and $n_{j}$ in Eqns. (37) and (38) represent the direction cosine of the corresponding vectors with respect to the $k$ and $j$-direction respectively. $t_{j}$ denotes the traction. We also should note the summation convention for the tensor in Eqns. (33)-(38). Green's function $G_{k j(i)}$ indicates the corresponding part of $G_{k j}$ associated with the fast longitudinal wave $(i=1)$ or slow longitudinal wave $(i=2)$, so that $G_{k 4(i)} \xi_{i} u_{i(i)}^{*}=G_{k 4(1)} \xi_{1} u_{k(1)}^{*}+G_{k 4(2)} \xi_{2} u_{k(2)}^{*}$. In Eqns. (28)-(32), by means of the parameters $a^{(1)}, a^{(2)}$ and $b$, the corresponding parts associated with the fast longitudinal wave, slow longitudinal wave, and transverse wave, can be readily identified respectively. The coefficient $c_{s}$ in Somigliana's integration Eqns. (33) and (34) are 1, because the buried source is in the interior of integration domain; $\mathrm{d} S$ is the integral element of the surface. The Green's functions are expressed as Eqns. (28)-(32). The integration domain in Eqns. (33), (34) and (35) is over free surface of the half-space.

\section{Numerical examples}

In the numerical examples below, the involved parameters are taken as $\lambda=1.29 \times 10^{7} \mathrm{~Pa}$ and $\mu=1.94 \times 10^{7} \mathrm{~Pa}$, the Poisson's ratio of soil skeleton $v=0.2$, $\alpha=0.83, \quad \beta_{0}=0.6, \quad \rho_{s}=2700 \mathrm{~kg} / \mathrm{m}^{3}, \quad \rho=\beta_{0} \rho_{f}+\left(1-\beta_{0}\right) \rho_{s}=1680 \mathrm{~kg} / \mathrm{m}^{3}$, $E_{w}=2.10 \times 10^{9} \mathrm{~Pa}, \rho_{f}=1000 \mathrm{~kg} / \mathrm{m}^{3}$. The concentrated vertical force is applied at the depth $h=2,2.5$ and $3 \mathrm{~m}$ respectively. The magnitude of force is fixed at $\mathrm{F}_{0}=1000$ N. Figs. 2-9 show the numerical results from our solutions, where the 
unit of the displacement is in millimeters, the unit of pore pressure is in $\mathrm{kPa}$, the unit for drainage flux is in Litre/s $\left((\mathrm{d} m)^{3} / s\right)$, and the dimensionless wave number is $k=p h / k_{\beta}$.

The drained and undrained soil displacements $u_{z}^{*}$ at fixed field point ( $r=1 m, h=0$ ) vs. dimensionless wave number $k$ are shown in Fig. 2, where we can find that the maximum value of soil displacement $u_{z}^{*}$ in the undrained condition is larger than that in the drained case. Fig. 3 illustrates that the undrained displacement of soil $u_{z}^{*}(r=1 m, h=0)$ vs. dimensionless wave number $k$, for the force being applied at different buried depths. Notably, the displacement $u_{z}^{*}$ decreases with increasing buried depth. Fig. 4 shows the drained displacement of soil $u_{z}^{*}(r=1 m, h=0)$ vs. dimensionless wave number $k$ for different depths of the force. Fig. 5 illustrates the drained and undrained pore pressure $p_{w} \quad(r=1 m, h=0)$ vs. dimensionless wave number $k$ respectively, where the maximum value of the undrained pore pressure is larger than that of the drained one. Fig. 6 illustrates the undrained pore pressure $p_{w}(r=1 m, z=0)$ vs. dimensionless wave number $k$ at different depths of the force where the pore pressure decreases with increasing source depth. Fig. 7 illustrates the drained pore pressure $p_{w}(r=1 m, h=0)$ vs. dimensionless wave number $k$ for different depths of the force. It should be noted that the pore pressure mentioned in this paper is an increment with respect to the atmospheric pressure. Fig. 8 illustrates the flux of drainage $(r=1 m, h=0)$ vs. dimensionless wave number $k$ for different depths of the force. The flux of drainage decreases with increasing source depth. While Fig. 8 is the result in the frequency domain, Fig. 9 shows the corresponding result in the time domain via the Fourier inverse. It is observed from Fig. 9 that the maximum flux of drainage at the field point $(r=1 m, h=0)$ is $0.05 \mathrm{~L} / \mathrm{s}$.

Vibroflotation in ground soil treatment makes use of the vibration of the vibroflot buried under ground to drain water. The vibration of the vibroflot is generally simulated by the impulse of sine function or the rectangular periodic function (Figs.10 and 14). Thus, the total response can be obtained by Duhamel's integral formula, i.e.:

$$
\mathrm{H}(t)=\int_{0}^{t} F(\tau) h(t-\tau) d \tau
$$

In Eqn (39) the unit impulse function $h(t-\tau)$ is the response of the displacement, pore pressure or drainage flux in time domain due to the action of a unit impulse of $\delta(t)$ in the poroelastic half-space. Hence, Duhamel's integration Eqn. (39) can be applied to Eqns. (33), (34) and (35) to obtain the response of displacement and pore pressure with respect to drainage via vibroflotation. 


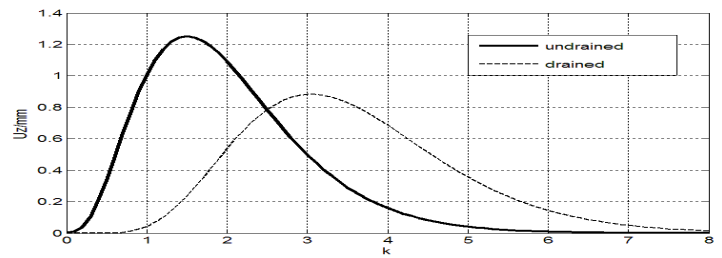

Figure 2: The soil displacement $u_{z}^{*}$ at $(r=1 m, h=0)$ in drained and undrained condition vs. dimensionless wave number $k$.

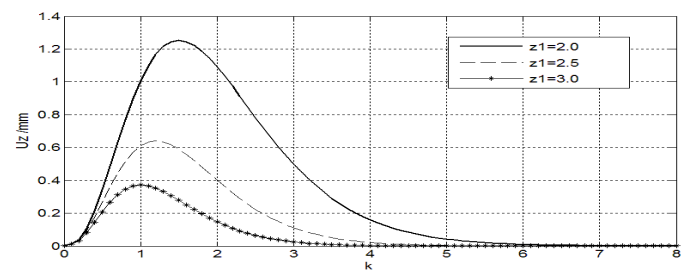

Figure 3: The undrained displacement of soil $u_{z}^{*}$ at $(r=1 m, h=0)$ vs. dimensionless wave number $k$ for different depths of the force.

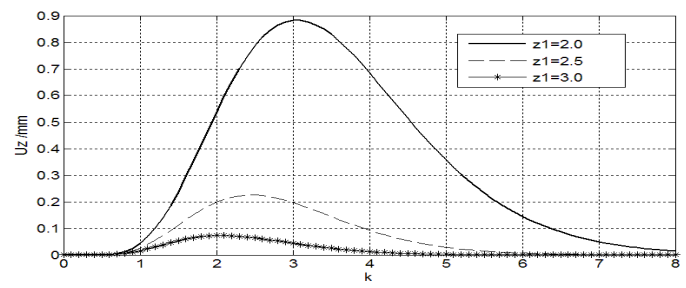

Figure 4: The drained displacement of soil $u_{z}^{*}$ at $(r=1 m, h=0)$ vs. dimensionless wave number $k$ for different depths of the force.

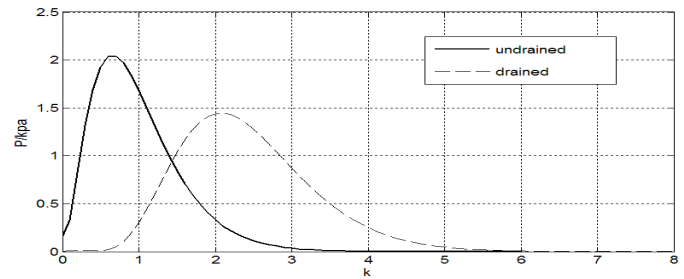

Figure 5: The porous pressure of soil $p$ at $(r=1 m, h=0)$ in drained and undrained condition vs. dimensionless wave number $k$. 


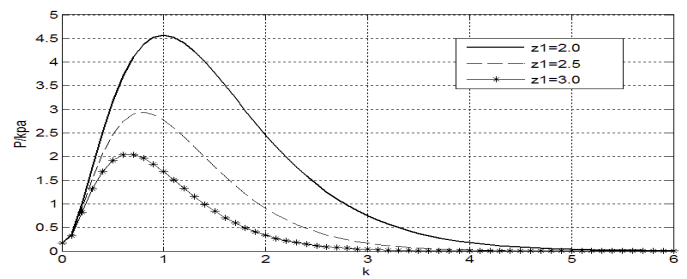

Figure 6: The undrained pore pressure of soil $p$ at $(r=1 m, h=0)$ vs. dimensionless wave number $k$ for different depths of the force.

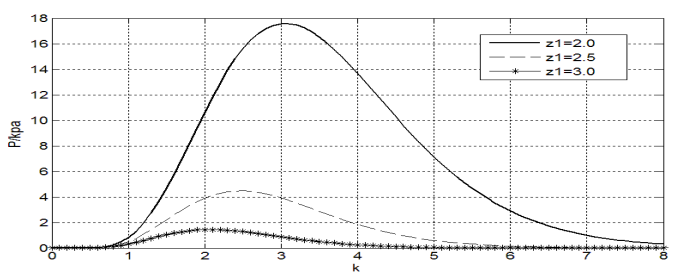

Figure 7: The drained porous pressure $p$ at $(r=1 m, h=0) \quad$ vs. dimensionless wave number $k$ for different depths of the force.

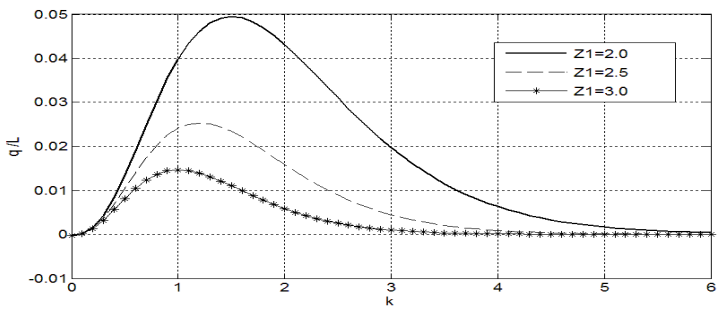

Figure 8: The flux of drainage at $(r=1 m, h=0)$ vs. dimensionless wave number $k$ for different depths of the force.

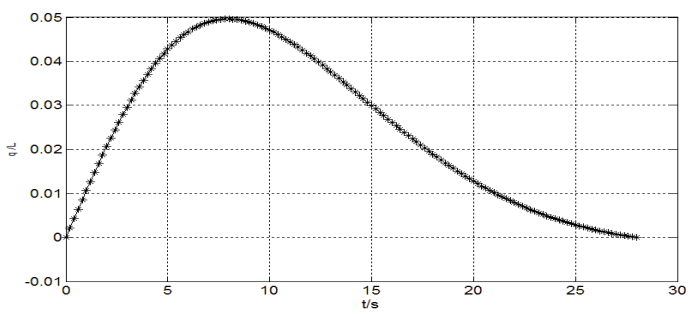

Figure 9: The drainage flux at $(r=1 m, h=0)$ vs. time when the force is applied at a depth of $2 \mathrm{~m}$ below the surface. 
We first assume that the vibration load of the vibroflot is stimulated by a sine function $F=F_{0} \sin (t)$ with the magnitude $F_{0}=10 \mathrm{KN}$ (Fig. 10) and it is located at a depth of $2 \mathrm{~m}$ below the surface. Shown in Fig. 11 is the drainage rate on the surface at $\mathrm{r}=1 \mathrm{~m}$ for a period. It is observed that the maximum drainage rate is $5.57 \mathrm{~L} / \mathrm{s}$. The drainage flux at the same period is shown in Fig. 12 with the maximum being $18.58 \mathrm{~L}$. Fig. 13 shows the corresponding result for $10 \mathrm{~min}$. where the maximum is $70.40 \mathrm{~L}$.

The vibration load of the vibroflot can be also stimulated by the rectangular periodic function with magnitude $F_{0}=10 \mathrm{KN}$ (Fig. 14) also located at a depth of $2 \mathrm{~m}$ below the surface. The drainage rate on the surface at $\mathrm{r}=1 \mathrm{~m}$ for a period is shown in Fig. 15 with the maximum rate being 5.67L/s. The drainage flux is shown in Fig. 16 for a period with the maximum being $15.18 \mathrm{~L}$ and Fig. 17 for $10 \mathrm{~min}$. with the maximum being $86.68 \mathrm{~L}$.

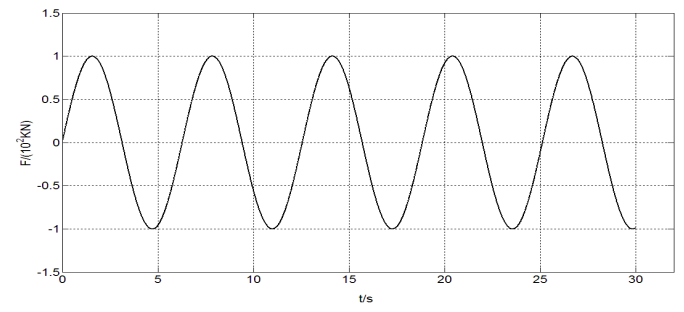

Figure 10: The sinusoidal periodic load for vibration simulation of vibroflot.

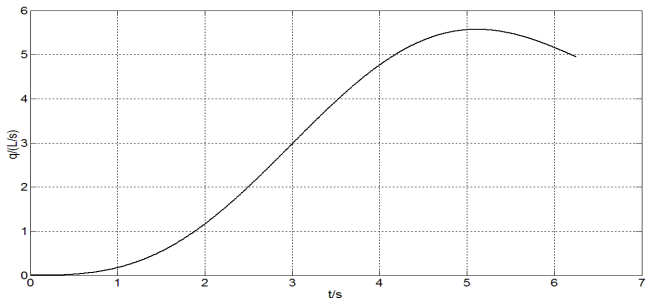

Figure 11: The drainage rate under the sinusoidal periodic load within a period.

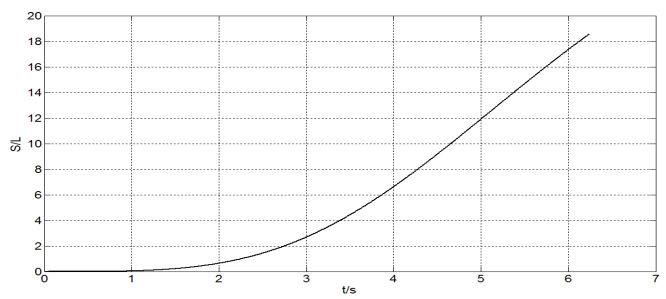

Figure 12: The drainage flux under the sine periodic load within a period. 


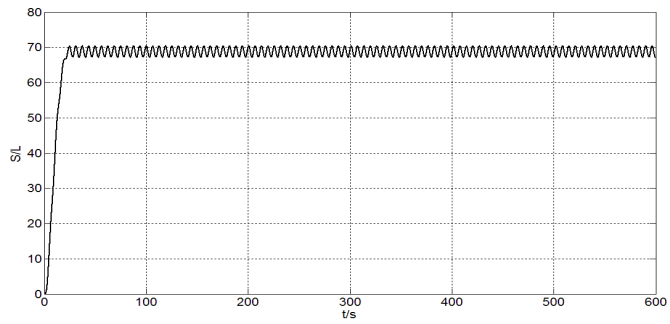

Figure 13: The drainage flux under the sine periodic load for 10 minutes.

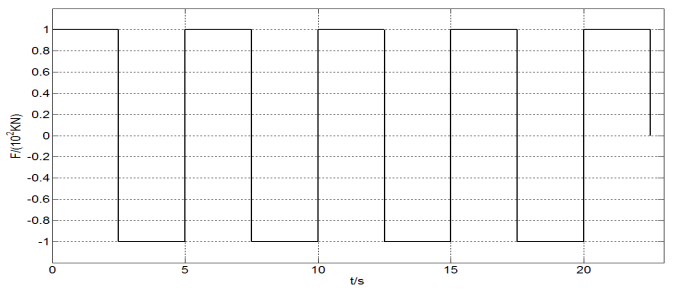

Figure 14: The rectangular periodic load for vibration simulation of vibroflot.

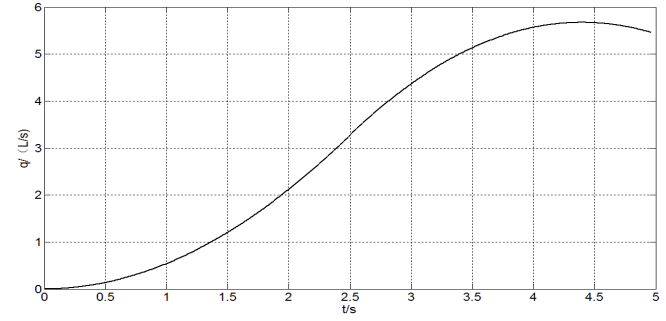

Figure 15: The drainage rate under the rectangular periodic load within a period.

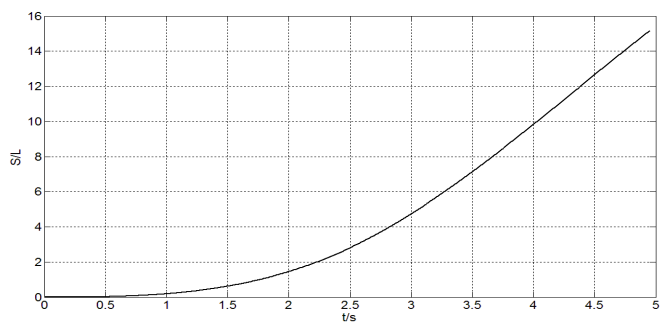

Figure 16: The drainage flux under the rectangular periodic load within a period. 


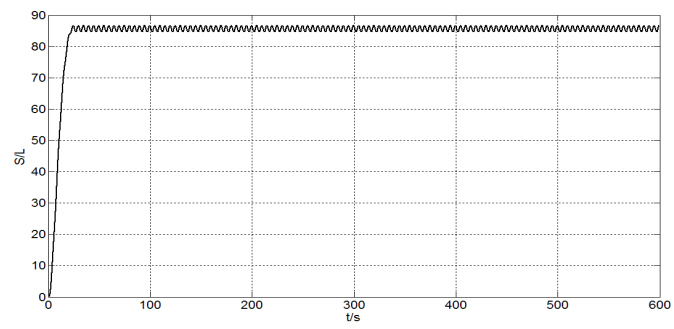

Figure 17: The drainage flux under the rectangular periodic load for 10 minutes.

\section{Conclusion}

The Green's functions of a poroelastic medium in the solid phase and fluid phase subjected to a buried load are derived and applied to estimate the drainagerelated issues for in saturated soil, with special application of vibroflotation in ground treatment. We point out that since the drained computation in this paper is derived by based on the Biot's equation and dynamic Darcy's law, the irreversible deformation effect of the vibroflotation is not accounted for. Additionally, the assumption on the axisymmetric deformation and further on neglecting the radial displacement may not be reasonable in ground treatment with vibroflotation. Nevertheless, the model presented in this paper may be useful in geotechnical engineering and geodynamics where drainage is involved.

\section{Acknowledgements}

We would like to thank Professor E. Pan of the University of Akron for reading through the article. We acknowledge the financial support from the National Natural Science Foundation of China (No. 11172268).

\section{References}

[1] Kaynia, A.M. and Banerjee, P.K., Fundamental solutions of Biot's equation of dynamic poroelasticity. International Journal of Engineering Science, 31, pp. 817-830, 1993.

[2] Lu, J.F. and Jeng, D.S., A half-space saturated poro-elastic medium subjected to a moving point load. International Journal of Solids and Structures, 44, pp. 573-586, 2007.

[3] Ding, B.Y., Song, X.C. and Yuan, J.H., The solution of Green function of fluid phase in two-phase saturated medium. Chinese Journal of Geophysics, 52(7), pp. 1858-1866, 2009.

[4] Biot, M.A., General theory of three- dimensional consolidation. Journal of Applied Phycology, 12, pp. 155-164, 1941. 
[5] Biot, M.A., The theory of propagation of elastic waves in a fluid-saturated porous solid. I, low frequency range. Journal of the Acoustical Society of America, 28, pp. 78-168, 1956.

[6] Biot, M.A., The theory of propagation of elastic waves in a fluid-saturated porous solid. II, high frequency range. Journal of the Acoustical Society of America, 28, pp. 91-179, 1956.

[7] Huang, Y. and Zhang, Y.H., Three-dimensional non-axisymmetric Lamb's problem for saturated soil. Science in China (Series E), 30(4), pp. 375-384, 2000.

[8] Philippacopoulos, A.J., Lamb's problem for fluid-saturated, porous media. Bulletin of the Seismological Society of America, 78, pp. 908-923, 1988.

[9] Jin, B. and Liu, H., Dynamic response of a poroelastic half space to horizontal buried loading. International Journal of Solids and Structures, 38, pp. 8053-8064, 2001.

[10] Cheng, A.H.D., Badmus, T. and Beskos, D.E., Integral equation for dynamic poroelasticity in frequency domain with BEM solution. Journal of Engineering Mechanics, ASCE, 117(5), pp. 1136-1157, 1991.

[11] Ding, B.Y., Tong, X.G., Chen, J. and Ouyang, M., The analyses for dynamic property of a single pile with drainage in the saturated soil. Chinese Journal of Geotechnical Engineering, 35(1), pp. 85-95, 2013.

[12] Ding, B.Y. and Yuan, J.H., Dynamic Green's functions of a two-phase saturated medium subjected to concentrated force. International Journal of Solid Structures, 48, pp. 2288-2303, 2011.

[13] Cheng, A.H.D, Ding B.Y. and Chen Z.L., Fundamental solutions of poroelasto-dynamics in frequency domain based on wave decomposition. dx.doi.org/ 10.1115/1.4023692, 2013.

[14] Chen, S.L., Zhang, J.M. and Chen, L.Z., Dynamic Green's functions of saturated soils subjected to the internal excitation. Chinese Journal of Geotechnical Engineering, 23(4), pp. 423-426, 2001.

[15] Chen, S.L., Chen, L.Z. and Pan, E., Vertical vibration of a flexible plate with rigid core on saturated ground. ASCE Journal of Engineering Mechanics, 133, pp. 326-337, 2007.

[16] Ding, B.Y., Dang, G.H. and Yuan, J.H., Lamb's integral formulas of twophase saturated medium for soil dynamic problems with drainage. Applied Mathematics and Mechanics, 31(9), pp. 1066-1074, 2010. 